\title{
Sensor Data Meets Social Networks Reflecting on Benefits in the Case of a Patient Room
}

\author{
Fabienne Kuhn, Andreas Spichiger, and Reinhard Riedl \\ Bern University of Applied Sciences, Bern, Switzerland \\ \{fabienne.kuhn, andreas.spichiger, reinhard.riedl\}@bfh.ch
}

\begin{abstract}
In a hospital, information exchange is essential to save lives and to prevent life-endangering mistakes. Information exchange is supported by a hospital information system (HIS). From a theoretical perspective, the deployment of an HIS is promising because it reduces errors and duplication of information. In practice, however, there are some major problems concerning the usage of such a system. One way to deal with these problems is introduced in this paper: the integration of sensor data into social media. The paper concentrates on the conceptual benefits and risks such an integration may generate. It focuses on the case of a patient room.
\end{abstract}

Keywords: Social media, sensor media, requirements engineering, ubiquitous computing, information provider, information access, Web 2.0, E-health.

\section{Introduction}

The information management in a hospital is often supported by a hospital information system (HIS) which addresses the various needs in a hospital by integrating most applications used there [10]. HIS provides some essential benefits, but there can still be major problems when using it on a daily basis. Benefits of using an effective HIS include information integrity, prevention of medication errors, reduction of transcription errors, duplication of information entries and report turnaround time [10], [12]. Problems arise in the following areas [33], [39], [30], among others:

Data recording and storage

- Immediate data access is required in a context where urgent cases are commonplace. Depending on the data storage solution, this requirement may not be met because data cannot always be recorded and stored in a timely manner. Sometimes not only the data storage but also the workflow does not allow instant recording of data. Concerns arise that information does not flow quickly enough during personnel changeovers, e.g. a change of shift.

- When a patient moves from one ward to another there are no techniques to move his data along effectively. This is because one organization unit cannot copy just one patient's data from another organization unit. Furthermore it is not easy to find old data about a newly arrived patient, for example when this person comes from an external domain. 
- Classic information systems are constructed for people who work on computers and nursing staff work with humans. The time taken to record data sometimes is longer than is allowed for in a nurse's job description. This leads to poor recording habits like copy/paste from other reports or shortening notes so they become unspecific. Thus wrong medication ${ }^{1}$ and wrong therapy can result.

Data view

- Doctors and nursing staff can lose the overview because there are many different screens where many details can be entered, which are not necessarily important but can be shown anyway.

- When data is not filtered and not declared, doctors and nursing staff may focus on specifics and do not see what others have written.

- Some approaches use alerts, reminders and warning messages to try to generate a specific view for each user. However, they can be sent regardless whether the information is relevant for the recipient or not. Thus the users tend to turn them off.

\section{Culture}

- Communication, which is very important in a hospital, is not simply about exchanging information. It is about generating effects; the sender wants the recipient to act. Furthermore, with direct communication the sender can find out how much the recipient understands, can correct a misunderstanding and can estimate and influence the recipient's actions. It is also about maintaining relationships.

We are interested in how user interfaces can be rebuilt as a smart system, focusing mainly on two problem areas. An important area concerning the problems mentioned earlier is the recording of data. It should be correct but not time consuming; it should support the staff but not interfere significantly with their workflow. A possible solution can be the deployment of sensors. Nevertheless it is risky to add more information into a system which already has several unresolved issues. The expectation is that it will not work. Let us follow this idea anyway: how should a system be rearranged to improve its usage? For a start there should be sensors which record the well-being of a patient and his whereabouts. Furthermore, medicines should be locatable to determine which medicine was given to whom. Another important problem area is the data view. The view should be composed user-and-situation-specific way without irrelevant data. Social media can provide those views. It also addresses another problem with HIS: data privacy. Social media offers the possibility to share selected data only with a selected group of people. Along with the previously mentioned requirements, which can be met either by sensor or social media, others can be met by the combination of both. For example the actors may comprehend the context and orient themselves better. This paper shows further possible conceptual benefits and risks concerning the idea of the combination of sensor and social media (SoSeMe) as a possible solution to the problems mentioned above. We have concentrated on the case of a patient room. This case was selected because a patient room provides a small and controlled environment and different stakeholders meet multifaceted challenges.

\footnotetext{
${ }^{1}$ See also [41].
} 
Fig. 1 gives an overview of the subjects raised thereby. Our research follows research guidelines according to Hevner [32]. In compiling the results we have mainly focused on the dimensions quality and economy. The paper is structured as follows: Theoretical basics and ideas are provided in chapter 2. Chapter 3 summarizes the case results and shows topics discussed for the patient-room case.

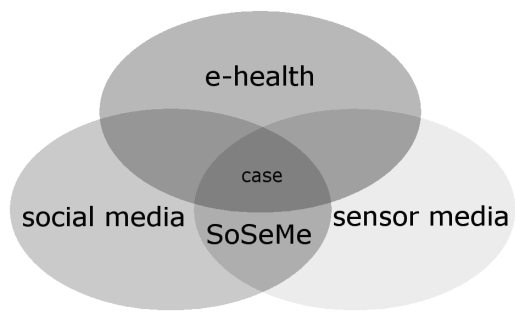

Fig. 1. Situation of the subject of this paper

\section{Related Research Work}

The idea of combining social and sensor media is not new. When users are requested to tag their posts with keywords, the benefits increase because it is easier to find posts related to specific topics [15]. An example here is Twitter, there the user can tag keywords by typing a "\#” at the beginning. This makes it easier for machines to find relevant related information for analysis. For example natural disasters are easier to detect and to monitor by mining the posts of social media users when they distinguish keywords like the name of the service which analyzes the data [16]. Areas where social and sensor networks can be linked effectively are shown in [36]. The article concludes that SoSeMe applications are more context aware. They are more deeply integrated into daily life and can act as an enabler for emerging services, e.g. mobile communication, internet, multimedia, etc. The framework in [37] describes communication in e-health primarily through social media. Those mentioned papers clearly indicate potential benefits. Even so, SoSeMe contrasts itself by researching in an area where data is mainly gathered through sensors, not people, and stored within the logic of social media. Furthermore the communication processes and rituals in a patient room are examined. With this approach, advantages in the limited setting and the intricacies of traditional communication are well understood.

\section{Data Processing}

Sensor and social media have different roles to play. In the following chapters their relevant roles are introduced for the case. This short theoretical background focuses on data processing: how data is acquired, stored and viewed. Furthermore it introduces topics inevitably connected with either social or sensor media in the context of a patient room.

\section{$2.1 \quad$ E-Health}

E-health focuses on the technical side of electronic media and adapts it to health care [17]. Health 2.0 uses networks of stakeholders like patients, doctors and scientists to 
personalize health care and promote collaboration [18]. It allows the patients to actively participate in their own healthcare through information and communication technology. In Fig. 1 Health 2.0 is the interface between e-health and social media.

Health networks may have the same problem as large enterprises: they are extremely complex and different parts often operate in silos [19]. This makes it hard to communicate across organizational units. However, if the flow of information between them stagnates, significant risks would be created for the patients. In addition, the non-sharing of information increases the risk of reacting too late to the spreading of infectious diseases. One solution to the latter problem is found in the Global Pandemic Initiative from 2006, where IBM offers several pieces of its healthcare technology portfolio to work with major worldwide public institutions [20]. Technologies offered include a framework to improve communication and collaboration, building a community of users which can tap into information as well as conducting research on influenza viruses. A solution for the former disadvantage may be found in patient centered electronic health records (EHR). EHR offers an overview of important medical data about a patient. The patient decides himself who gets access to his data.

\subsection{Sensor Media}

Sensors record their surroundings on demand, regularly or permanently within a time period. They can determine the position, movement and further attributes of an object at a specific time. They can also detect pressure to find out the weight of an item or detect the movements of a person during the day, categorizing them into different activities [34]. Sensors can be put together into a network in which they communicate with each other [8], [25]. The combination of networked sensors opens new possibilities and more attributes can be measured. Correlation allows a more precise acquisition of an object's state and it is possible to see the bigger context. In the patient room the combination of sensors in a network can, for example, detect that the patient fell in the wet room at 12:00 p.m. Sensor media record an event but with sensors alone there is no reaction. How does the nurse know a patient has had a fall?

\subsection{Sensor Deployment in Healthcare}

The deployment of sensors in healthcare has five main application areas [21]: process controlling and documentation, localization, personalized patient medicine and identification, monitoring of measured data and protection against fake medicine.

In process controlling, the sensor technology can facilitate processes and organize them more efficiently based on data collected through sensors. This can optimize product logistics and cost control. Processes concerning documentation can also be simplified, which results in timesaving and reduction of mistakes. Through a better localization of personnel, materials and devices, search time can be reduced and information about the inventory can be provided. One of the most frequent mistakes is the dispensing of the wrong medicine. Insufficient information about the exact kind, amount and time the medicine was administered is one of the causes. A personalized RFID tag can prevent such mistakes, because with this, all information can be allocated to a specific patient. So it can be ensured that the right patient gets the right 
medicine. The measurement and monitoring of vital signs is relevant for product logistics. The collected data is transferred into an emergency system that raises an alarm when a specific range is exceeded. Protection against counterfeit medicine is an issue whose relevance is increasing constantly. RFID tags enable the definite identification of medicines and permanent, gapless data alignment. This simplifies the distinction between fakes and originals.

\subsection{Social Media}

With social media people can share data, view data and use others' data. Users have the possibility of sharing data publicly or with a predefined specific group of people [11]. Shared data have different levels of quality. They can even be faked to generate a specific impression, for example to compete or to impress. Most social media further provide individual views of specific situations ${ }^{2}$. This means the information about a situation is selected to create specific, person-centered perspectives.

Social media can also be seen as a recorder for time-dependent distributed states [5]. The concept of the time line increases the quality of the data on social media because each data item gets an exact timestamp and orders data chronologically.

Social media are also able to acquire data about the relationship between persons, or persons and items, or even items and items, based on specific data which are uploaded by the people themselves [13]. The possibility to link data, e.g. by tagging photos with the persons present on them, has proven to be an important tool for relationship management. One of the more recent trends is that people tend to share and comment on data which they find important, rather than uploading data themselves [31].

With social media, the patient could inform the nursing staff that he fell down in the wet room at 12:00 p.m. But how does he do that when he is unconscious?

\subsection{Social and Sensor Media Fusion}

In SoSeMe, data which is collected and joined by sensor media are stored in and distributed by social media. Data is provided by the involved actors/stakeholders who write statements into social media, by the sensor network, and through the hospital information system. The social media set-up plus the attribution of metadata help to contextualize the information, customized for those who access it. This makes the information pick-up much easier and opens a whole new experience in data handling. In particular, both missing the pick-up of relevant information and giving wrong interpretation to the accessed data become less likely. While the set-up particularly addresses humans, it could equally be employed to steer actuators based on artificial intelligence.

Provided that the patient shared his data with nursing staff and that the patient room is equipped with sensors, the data collected when he fell down in the wet room at 12:00 p.m. can be stored in social media and the nursing staff will be informed and can provide immediate help.

\footnotetext{
${ }^{2}$ For further information about an instrument how this could work see https://developers.facebook.com/docs/technical-guides/fql/
} 


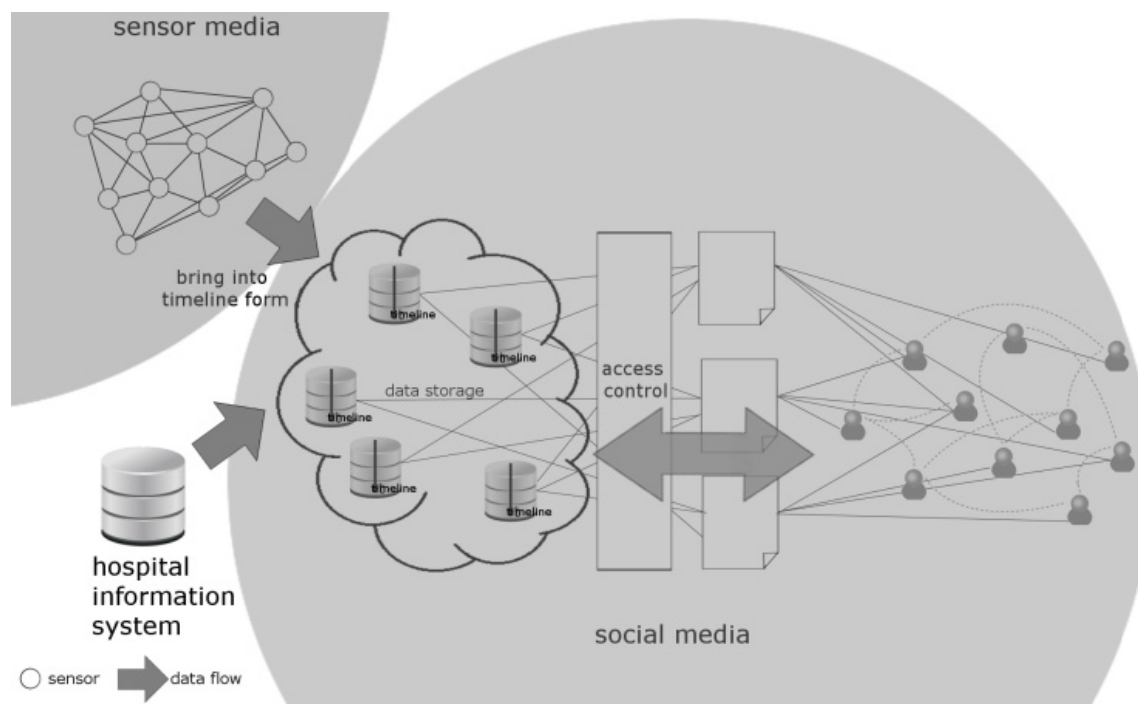

Fig. 2. Interplay between social and sensor media

\section{Case Patient Room}

\subsection{General Scenario}

The activities in the patient room include people with mixed attributes like patients, visitors and health professionals who communicate with one another and interact with things. There are lots of situations when communication is of critical importance for the health of the patient. The intricacies and problems of this communication are well understood. On the one hand, the need for high quality integration of information is obvious; on the other hand a lot of this information concerns the privacy of patients, which has to be protected. A lot of different kinds of sensors may be placed to observe the room with all its content almost fully, but there is also a lot of social context information being created outside, which also plays a key role.

For a better understanding of the current situation, we have conducted qualitative, semi-structured interviews with the staff in hospitals. Furthermore the processes in a hospital were observed. Those empirical assessments were made according to Pohl [22] in the hospitals Spital Zentrum Biel, Spital Aarberg and Inselspital Bern. Those hospitals were selected to have a comparison between a small hospital which is typically equipped, one that has recent medical equipment as well as infrastructure and a large university hospital. The empirical assessments where focused on the available equipment, available information, nursing and information processes. To evaluate the results, the software MaxQDA $10^{3}$ was used. Parallel to and based on the empirical assessments we have determined three specific indicative scenarios for investigation (cf. chapter 3.2), for which we then have identified the optative scenarios and the

\footnotetext{
${ }^{3}$ For further information about this software see http: // www . maxqda. de
} 
related requirements also according to Pohl. This has yielded ideas about how to conceptually optimize patient rooms in the future, which we explain in chapter 3.3. Then we have built a prototype of a future patient room equipped with a sensor network.

Different prototype implementations were used to find out what is technically possible. Thus another scenario was evaluated. Active sensors were used to identify doctors and nursing staff, patients and medicine. The nurse carried a small device which showed information relevant to the room s/he was in. When the nurse had the wrong medicine for a patient, the device would give a warning. The drawback here is that the prototype reacted really slowly, meaning that the sensor network has to be optimized. This paper does not go deeper into the results generated by the prototype, because they only provide a basis for further project ideas.

\subsection{Patient Room Today}

We looked at the situation of information management in three hospitals in and around Bern, Switzerland. Each had differently equipped patient rooms. However, in all cases there was a paging system, an intravenous pole, an intravenous bag, an infusion set, an Infusomat and a bed.

With the patient paging system the patient can call for a nurse, which simplifies communication between the patient room and the nurses' room. This system consists of a button or a string near the bed, the shower, the toilet and the eating table. When a patient needs help, he pushes the button. Then a lamp turns on beside the door. In the corridor and nurses' room is a display which shows in which room the button was pushed. After entering the room, the nurse turns off the lamp beside the door, so other nurses know that this patient is already being attended to. The nurse can also signal an emergency through this system. Then the door lamp shows the letter N (for 'Notfall', which is German for emergency).

Intravenous bags contain the infusion solution. The name of the infusion and additional information about the content and context (e.g. the nurse's signature) of the bag are written on the bag.

Infusomats $^{4}$ are electronic pumps which ensure the supply of liquid drugs during longer periods. Each Infusomat has a controlled hose clip with which an exact dosage for the infusion solution per interval is possible. They show the total amount of infusion solution which has already passed through, how many milliliters per hour should be administered and how many milliliters have already been administered. Some Infusomats have an integrated sensor which counts each drop. When all of the infusion solution has passed through, the device reports the end of the infusion.

Infusions can cause problems like vein inflammation which cannot be detected immediately. Furthermore it is sometimes not possible to determine whether the intravenous catheter is set right into the vein, which could inhibit blood flow.

In several scenarios the bed plays a central part; from here the patient interacts with his environment. As a minimum, the height and the angle of the bedhead are adjustable. In the bed the patient can be transported. In most cases the bed can be extended.

\footnotetext{
${ }^{4}$ Infusomat is a trademark of B. Braun Melsungen AG.
} 
In a patient room there are three main groups of persons: patient, staff and visitors. The patient suffers from illness or the consequences of an accident. He is identified through his name and forename which are written on the bed and the wardrobes. In the researched context, the staff mainly consists of the doctors and nursing staff, which provide services to the patients. They wear a badge on which relevant metadata about their person is written. The doctors prescribe drugs and enter the prescriptions into the hospital information system.

Finally, time management is an important issue. For example there is a schedule for the visits of the chief physician. The use of digital information processing systems is still rather limited. A lot of the information processed only exists in the heads of the staff and is communicated mostly in face-to-face meetings.

\subsection{Patient Room in the Future}

In the following paragraphs the vision of the interviewed persons about the future patient room is summarized and adapted to the main aspects for this case.

The patient room of the future is a smart system and in this case is called smart patient room system (SPRS). It monitors and controls the patient's situation because it has the best access to all relevant information. SPRS is the specific product of SoSeMe for the case patient room.

The patient page system is automated through new technologies. The HIS detects critical situations on the basis of sensor data and sends for a nurse who herself has an individual device which shows her relevant patient data.

The intravenous pole recognizes infusion and drugs. The SPRS compares these with the patient data. If there is a prescription for the correctly identified patient, it starts the administration of the infusion. If any error occurs, the system will warn the nurse about the danger.

The catheter of an infusion could be improved by adding a sensor which can determine if it is properly in the vein or not.

The bed is intelligent, but still easy to use and comfortable. It records the patient's vital signs, body temperature, blood pressure and weight repeatedly and sends the data through a communication channel to social media storage.

Consumables like infusion bags and medicine are equipped with RFID-tags.

\subsection{Specific Scenario Versions}

Throughout the interviews, with nurses working at different hospitals and an M.Sc. class of nurses, we assessed the situation in standard patient rooms and the problems arising because of lack of easily accessible information. Out of the large number of possible scenarios three were selected. The specific scenario 'Infusomat' was selected because it addresses major issues (identifying the patient, dispensing of the wrong medicines) and also includes an actuator, as well as the necessary sensors. The specific scenario 'Vital Signs' was selected because this scenario happens frequently and integration of information flow would improve the work significantly. The specific scenario 'Visitors' was selected to show how people get involved in information 
diffusion in a patient room. The goal of the selection was to have a minimal set of scenarios that allow all the important benefits and risks to be demonstrated. After the description of today's process of the indicative scenarios, they were optimized as optative scenarios to fit in the future patient room, as shown below. The prototype of the future patient room was then built for optimal support of these scenarios.

\section{Infusomat}

A patient is suffering from a chronic lung disease and needs an analgesic. His nurse orders an infusion over the SPRS by voice command through her headset. She arranges the printing of an RFID tag, which she uses to get the analgesic and infusion from the medicine cabinet. She sticks the tag on the infusion bag and adds the analgesic to the infusion. The infusion bag is attached and integrated to the smart infusion booth (SIB), an intravenous pole with built-in electronics. The SPRS reads both SIB and RFID tag, identifies the patient and checks for a match between the data and the prescription. The SPRS gives the order to SIB to adjust. The SIB gives the signal for the infusion. The nurse attaches the infusion to the patient and starts the SIB. After an hour, when the infusion is finished, the SIB sends a signal to the SPRS that the infusion is finished. The SPRS informs the nurse with a voice mail. Then it turns itself off. The nurse removes the infusion.

\section{Vital Signs}

During the first four hours after an operation, the patient's vital signs, like blood pressure, temperature, oxygen saturation and wakefulness are determined and added to his electronic health records (EHR) every half an hour. In addition, the nurse asks the patient about nausea, vomiting, pain and the urge to void. The nurse appends the collected data to the EHR with the aid of a mobile device. The data can be accessed by doctors via social media, allowing better treatment if the patient has agreed to share EHR with them.

\section{Visitors}

There are two patients in the room. One has company from his wife, the other from a coworker. The SPRS identifies the wife and the coworker by video records. If the visitors wish it, SPRS creates a digital relationship between those two and connects this with various additional data, such as the nature of the relationship between patient and visitor (e.g. the relation between relatives and patients may be stronger than the one between coworkers and patients).

\subsection{Potential Benefits}

The analysis of the three specific versions of the more general patient room scenario have shown different areas where the use of sensor data and the providing of the necessary information in the right form by social media can produce benefits, mainly in the areas of quality and economy. A summary including stakeholders' perspectives is shown below. Note that the stakeholder list is not complete. Other stakeholders outside the patient room which can benefit from SoSeMe include hospitals, the pharmaceutical industry, health insurers, researchers and governments. 


\section{Patient and Medicine Identification Improves Medicine Management}

- The system has the possibility to align the medicine and patient data.

Nursing - Nurses are prevented from making mistakes, because the system

Staff warns if someone tries to give the wrong medication.

- They can provide the right amount of medicine in a timely manner.

Patients - The patients get the correct treatment.

\section{Automated, Real-time Information Exchange around the Clock Improves Communication and Data Flow}

- Information and experiences with specific situations can be exchanged and shared free of barriers and media disruption.

- Communication is quick, easy and location- as well as time-independent.

- The system supports the nursing stuff to explain the patients' health in an understandable but correct way.

- Stakeholder interactions are improved by the exchange of opinions based on data.

- There is the possibility of location- and time-independent patient monitoring.

Nursing - Immediate information access in case of an emergency.

Staff - The insight of the patient's relatives can systematically be collected.

- They can improve checklists about symptoms, which are relevant for the classification of appropriate therapy.

Patients - The patients can participate in the decisions doctors render.

- They get more secure treatments.

- They can move more freely.

Relatives - They can seek support from other relatives.

\section{Automatic Information Acquisition Improves Stakeholder Management}

- It is easier to perform analysis based on information about stakeholders. The system allows an easier collaboration.

Nursing - Staff can be deployed and scheduled more effectively.

Staff - Nurses can organize their nursing services better.

Patients, - They get faster and more correct information.

Relatives

\section{Sensor Improve Workflow Facilitation}

- Time-consuming and complex tasks can be facilitated.

Nursing - Data input through headsets instead of typing.

Staff - Nurses can organize their nursing services better.

Patients - Record symptoms at moment of event. 


\subsection{Potential Risks}

The cooperation of patients is needed for the success of the idea of SoSeMe. However, due to data protection concerns, patients may tend to try to hide things from their doctors [34] and manipulate sensors on purpose. This strategy may have consequences for the quality of the treatment. Moreover, doctors too may have the impression that their work would be checked more than before and they would have to justify themselves. Because of this feeling, doctors may tend to concentrate on the awareness of third parties rather than their patient's wellbeing.

With the social media in SoSeMe the patient is the data owner and can, as with the EHR, decide who gets access. The idea of SoSeMe depends on the patient's willingness to share his data and also his trust in technology. The amount of information available for the stakeholders increases with the integration of social and sensor media, meaning that analysis of this machine-sourced data could be invalid unless the significance of each piece of information is considered first. This argument is based on the assumption that people weight the information they communicate through speech by variation of pitch level and volume. Also the impression may arise that knowledge about the context is fully available and the importance of a piece of information is weighted differently by different stakeholders. Decisions can be made based on this impression. Furthermore, the nursing staff and doctors have to decide how much they want to know from their patients because of information processing costs and the risk increases with too much information.

Filters and access control may not work reliably or stakeholders abuse their information access. Therefore it is possible that people get access to data which they should not see. Furthermore, should patient data transfer not be encrypted as required by law [35], the risk of unauthorized data access and data abuse would increase. The usability of input devices is a further aspect which influences the quality of information in the system and the costs of information processing. As long as information has to be typed in rather than told to somebody, more time is needed or less information can be recorded. On the other hand, audio records cannot be processed optimally by machines. This introduces new areas of error.

\subsection{Discussion}

A future patient room should be as smart as possible so that the patient has more free space and can communicate with fewer barriers. The room should offer the nursing staff facilities within their workflow as well as location- and time-independent communication for patients, staff and visitors.

The combination of social and sensor media can also be used as an emergency system, because it can respond in a timely manner [23]. No matter how promising the integration of sensor data from a patient room into a social network is, it depends on the patient's maturity and sense of responsibility to himself. The interviews we conducted show that the integration of sensor technology can improve workflows. However, the interviewees also made clear that the resulting free capacity would be best used to increase the social interaction between nursing staff and patients and not cost cutting. 
The main challenge faced by SoSeMe is data privacy. Interviewees from a technical department declared they would never publish health relevant data. This statement is comforting regarding privacy protection but many people already use online forums or social media to seek help for their illnesses [1]. We analyzed the relevant laws in Switzerland. The nursing staff is not allowed to distribute personal data without the permission of the patient ${ }^{5}$. They must not give access to third parties without the permission of the patient ${ }^{6}$ and it is forbidden even to process the data unless the patient agrees to it first ${ }^{7}$. The patient is the data owner. This means the nursing staff has to be given access to this data and the patient decides who can access that data. With this, the main challenge faced by SoSeMe may be the main benefit, because SoSeMe permits the patients exactly what is stated by the law: an instrument with which they can act as data owner and easily give permission to organizations or persons to access data, process data or deny access altogether. Furthermore, through strict integration of social and sensor media data, privacy in systems can even be improved because violations of data privacy can be tracked.

SoSeMe includes the benefits of the patient's dossier $\left(\mathrm{EHR}^{8}\right)$ and enriches it. In today's e-health concepts, it is envisioned that patients will take more responsibility for their own dossiers [26], with hospitals providing added value to their patients by supplying their personal health data in a suitable way. Each patient then has the possibility to share his EHR with whomever, whenever and wherever they want, which includes non-sharing as well. The patient may share data related to their current visit, or if necessary, up to all of their recorded history, as their care requires.

The patient's room situation in a SoSeMe setting is a very complex system. The requirements elicitation on the basis of three rather simple indicative scenarios, defining the indicative information model, and then elaborating the corresponding operative scenarios and the future information model was shown to be a very direct way to define the future system. The applied requirements elicitation techniques in combination with stakeholder interviews proved to be a very effective method. In combination they are appropriate for a discussion on the high abstraction level of potential directions of future systems.

\section{Conclusion and Further Activities}

Our research has shown that the integration of digital social media and of sensor networks offers a rich set of options for improving the communication taking place in and around a patient room. At the same time it has revealed significant risks if such an integration is not handled with care. In addition, it may be expected that such experiments would face significant opposition and ethical concerns. Nevertheless the combination of social and sensor media can provide a possible solution to problems arisen in the deployment of a HIS.

\footnotetext{
${ }^{5}$ Related articles are amongst others SR 101 Art. 13, SR 235.1 Art. 1 and 35

${ }^{6}$ Related article is SR 235.1 Art. 8

${ }^{7}$ Related article is SR 235.1 Art. 17

${ }^{8}$ For further information on a patient dossier in Switzerland see http: / /www. evita.ch/en/home/.
} 
Potential benefits include the deployment of SoSeMe within the patient room, which may reduce wrong medication; patients can be monitored in real time and also be treated immediately in case of an emergency. Furthermore, medicine and personnel management can be improved, and workflow facilitated. Participation of patients in decision making can also be supported. Moreover, the idea of time line and collaboration can be a benefit for worldwide research, because with social media it is easier to connect locally independent information. Note that nurses' responsibilities are not being delegated to machines. Machines only act as support.

Since these benefits are so important, we have decided to continue our research and to investigate the specific sub-scenarios described in more depth. The patient room can further be equipped with more sensors such as video, audio and wireless sensor systems, which would allow, for example, location and identification of objects. In the patient room this could be used for checking the inventory [27], [19]. To find out what the patient ate a photo can be taken; to find out how much he ate, the food can be weighed. Zooming out of the patient room, in the future we shall look further at the hospital context as a whole or even into larger medical systems, such as the healthcare of a specific region.

Acknowledgements. The first thanks go to Emine Seker, Nicola Schwery and Michael Ferreira who provided important content for this project. Further thanks go to Ross Bennie, Alessia Neuroni and Thomas Jarchow, who reviewed this paper.

\section{References}

[1] Chun, S., MacKellar, B.: Social Health Data Integration using Semantic Web. In: SAC 2012, Riva del Garda, Italy, March 25-29, pp. 392-397. ACM, New York (2012), http: / /dx.doi.org/10.1145/2245276.2245351

[2] Bry, F., et al.: Digital Social Media, Dagstuhl Manifesto. University of Munich, Germany (2010), http: / /www.pms.ifi.lmu.de/publikationen/PMS-FB/ PMS-FB-2010-7/PMS-FB-2010-7-dagstuhl-manifesto.pdf

[3] Vera, R., Ochoa, S.F., Aldunate, R.G.: EDIPS: An Easy to Deploy Indoor Positioning System to support loosely coupled mobile work. Personal and Ubiquitous Computing 15(4), 365-374 (2011), http: //dx.doi.org/10.1007/s00779-010-0357-x

[4] Kamei, K., et al.: Tagging Strategies for Extracting Real-world Events with Networked Sensors. In: ICMI 2007, Nagoya, Japan, November 15, pp. 35-42. ACM, New York (2007), http: / / dx.doi .org/10.1145/1330588.1330594

[5] Sawyer, T.: Facebook Timeline. Rough Notes 155(66), 68-69 (2012), http: //search.proquest.com/docview/1010360195?accountid=15920

[6] Mullender, S.: Distributed Systems. ACM Press, United States of America (1993)

[7] Bantel, M.: Messgeräte-Praxis, Funktion und Einsatz moderner Messgeräte. Hanser Verlag, München Wien (2004)

[8] Dargie, W., Poellabauer, C.: Fundamentals of wireless sensor networks: theory and practice. John Wiley and Sons, Hoboken (2010) 
[9] Swedberg, C.: Michigan Researchers Develop RFID-based Sensors to Measure Physical Activity. RFID Journal (2010),

http: //www.rfidjournal.com/article/view/7884/

[10] EHR Scope: Hospital Information Systems (HIS) (2013),

http: / /www. emrconsultant.com/education/

hospital-information-systems

[11] Luo, W., Liu, J., Liu, J.: An Analysis of Security in Social Networks. In: DASC 2009, Chongqing, China, December 12-14, pp. 648-651 (2009),

http: / /dx.doi.org/10.1109/DASC.2009.100

[12] Watson, L.: Woman dies after replacement nurse gives her wrong drug during labout dispute. Daily Mail, September 26 (2011),

http: //www.dailymail.co.uk/news/article-2041884/

Woman-dies-replacement-nurse-gives-wrong-drug-labour-

dispute.html\#axzz2KCPIH9Of

[13] Yang, C.C., et al.: Identifying Implicit Relationships between Social Media Users to Support Social Commerce. In: ICEC 2012, Singapore, Singapore, August 07-08, pp. 41-47. ACM, New York (2012),

http: / /dx.doi.org/10.1145/2346536.2346544

[14] Sharp, J.: A Look at Social Media in Health Care - Two Years Later. iHealthBeat, May 17 (2012), http: / /www. iheal thbeat.org/perspectives / 2012 / a-look-at-social-media-in-health-care-two-years-later.aspx

[15] Nagarajan, M., Sheth, A., Velmurugan, S.: Citizen sensor data mining, social media analytics and development centric web applications. In: WWW 2011, Hyderabad, India, March 28-April 1, pp. 289-290. ACM, New York (2011), http: / /dx.doi.org/10.1145/1963192.1963315

[16] Aulov, O., Halem, M.: Human Sensor Networks for Improved Modeling of Natural Disasters. IEEE 100(10), 2812-2823 (2012),

http://dx.doi.org/10.1109/JPROC.2012.2195629

[17] Della Mea, V.: What is e-Health (2): The death of telemedicine? J. Med. Internet Res. 3(2), e22 (2001), http: / /dx.doi .org/10.2196/jmir.3.2.e22

[18] Bos, L., et al.: Patient 2.0 Empowerment. In: SWWS 2008, pp. 164-167 (2008), http://science.icmcc.org/2008/07/24/patient-20-empowerment

[19] Becker, E., et al.: A wireless sensor network architecture and its application in an assistive environment. In: PETRA 2008, Athens, Greece, July 15-19, Article No. 25. ACM, New York (2008), http: / / dx.doi.org/10.1145/1389586.1389616

[20] Loughran, M.: IBM, Public Health Groups Form Global Pandemic Initiative, Goal is to Identify, Map Pandemic Outbreaks and Better Target Vaccines. IBM News (May 2006), http: / / www-03.ibm.com/press/us/en/pressrelease/19640.wss

[21] Rost-Hein, M., Japs, S.: RFID im Gesundheitswesen, Die Anwendungsbereiche. Informationsforum RFID e.V. (2007), http: / / www. info-rfid.de/info-rfid/ content/e107/e127/e242/rfid_im_gesundheitswesen_ger.pdf

[22] Pohl, K.: Requirements Engineering. Fundamentals, Principles, and Techniques. Springer, London (2010)

[23] Kuehn, A., et al.: Interoperability and Information Brokers in Public Safety: An Approach toward Seamless Emergency Communications. Journal of Theoretical and Applied Electronic Commerce Research 6(1), 43-60 (2011), http://dx.doi.org/10.4067/S0718-18762011000100005 
[24] Atzori, L., Iera, A., Morabito, G.: The Internet of Things: A survey. Computer Networks 54(15) 2787-2805 (October 2010), http://dx.doi.org/10.1016/j.comnet.2010.05.010

[25] Wilson, A.D.: Human-Computer Interaction Handbook, Fundamentals, Evolving Technologies and Emerging Applications, pp. 177-199. CRC Press (2007), http: / / dx.doi.org/10.1201/b11963-10

[26] Van Noordende, G.: Security in the Dutch Electronic Patient Record System. In: SPIMACS 2010, pp. 21-32. ACM, New York (2010), http: / /dx.doi.org/10.1145/1866914.1866918

[27] Neumann, J., et al.: Integration of audiovisual sensors and technologies in a smart room. Personal and Ubiquitous Computing 13(1), 15-23 (2009), http: //dx.doi.org/10.1007/s00779-007-0172-1

[28] Aijaz, F., Chaudhary, A., Walke, B.: Mobile Web Services in Health Care and Sensor Networks. In: ICCSN 2010, pp. 254-259 (2010),

http: / /dx.doi.org/10.1109/ICCSN. 2010.42

[29] deBrankart, D.: Swiss ePatient Day - Opening Speech (2011), http: / / my.brainshark.com/ Swiss-ePatient-Day-Opening-Speech-122780377

[30] Wojciechowski, M.: Distributing and Replicating Data in Hospital Information Systems, http: //citeseerx.ist.psu.edu/viewdoc/ download?doi=10.1.1.80.4695\&rep=rep1\&type=pdf

[31] Burkhard, S., Schorre, S.: Ich weiß was du letzten Sommer gelesen hast - E-Reader und die Implikationen für den Datenschutz. In: Schweighofer, E., Kummer, F. (eds.) Europäische Projektkultur als Beitrag zur Rationalisierung des Rechts (Österreichische Computer Gesellschaft), IRIS 2011, pp. 35-44 (2011)

[32] Hevner, et al.: Design Science in Information Systems Research. MIS Quarterly 28(1), 75-105 (2004), http: / / em.wtu. edu.cn/mis / jxkz/sjkx.pdf

[33] Marion, J.B.: Hospital information systems: perspectives on problems and prospects, 1979 and 2002. International Journal of Medical Informatics 69(2-3) (2002), http: / /dx.doi.org/10.1016/S1386-5056 (02) 00098-9

[34] Denzler, L.: Das Arzt-Patienten-Verhältnis im digitalen Zeitalter. Unsere Gesundheitsdaten im Netz, eHealth Publifocus und Elektronisches Patientendossier TA-P10/2008 p. 12 (2008)

[35] Bosshard, G., et al.: Rechtliche Grundlagen im medizinischen Alltag, Ein Leitfaden für die Praxis. SAMW und FMH, Switzerland, pp. 80 (2008)

[36] Breslin, J.G.: Integrating Social Networks and Sensor Networks. In: W3C Workshop on the Future of Social Networking (2009)

[37] Stelle, R.: Social Media, Mobile Devices and Sensors: Categorizing New Techniques for Health Communication. In: ICST Fifth International Conference on Sensing Technology, pp. 187-192 (2011), http: / /dx.doi.org/10.1109/ICSensT.2011.6136960

[38] Ash, J.S., Berg, M., Coiera, E.: Some Unintended Consequences of Information Technology in Health Care: The Nature of Patient Care Information System-related Errors. J. Am. Med. Inform. Assoc., pp. 104-112, http://dx.doi.org/10.1197/jamia.M1471

[39] Bates, D.W.: Using information technology to reduce rates of medication errors in hospitals. BMJ 320(7237), 788-791 (2000) 